\title{
ETEC Vaccine between Reality and Expectations
}

\section{Nasr-Eldin Mohamed M. Aref*}

Department of Animal Medicine, Faculty of Veterinary Medicine, Assiut University, Assiut, Egypt

Strains of enterotoxigenic Escherichia coli (ETEC) have been recognized as the first leading cause of neonatal diarrhea and the second major cause of infant mortality in developing countries [1-2]. Insufficient data exist, but conservative estimates suggest that ETEC strains are incriminated as the cause of 280-400 million diarrheal episodes per year in children under 5 years of age, resulting in 300,000 to 500,000 deaths. ETEC is also the most common cause of travelers' diarrhea, being responsible for one-third to one-half of all diarrheal episodes in travelers to Africa, Asia and Latin America [3].

The main hallmarks of this type of bacteria are expression of more than one colonization factors (CFs) used for attachment to host intestinal cells and elaboration of one or more enterotoxins [4]. To date, 25 distinct CFs have been identified [5] and 2 major enterotoxins, the heat-stable (ST) and heat-labile (LT) enterotoxins. Both toxins elicit net secretion of ions and water, resulting in profuse watery diarrhea and potentially fatal dehydration.

The antigenic diversity of these strains (enterotoxins/colonization factors (CFs) combinations) accounts for the high prevalence of ETEC-induced diarrhea in the endemic areas and poses challenges for development of a broad-spectrum ETEC vaccine [5]. Currently, there is no licensed vaccine that confers a broad protection against the heterogeneous array of ETEC strains although recent donor investments from the USA (Bill \& Melinda Gates Foundation) and Europe (STOPENTERICS, GLOBVACEntVac) have reinvigorated the efforts on vaccine development [6].

A variety of strategies have been pursued in attempts to develop a broad spectrum ETEC vaccine. Prior studies suggest that liveattenuated or inactivated whole-cell vaccines may be viable options if safety and immunogenicity issues can be overcome. Promising data in this regard were reported from two recently completed Phase IIb immunization challenge studies evaluating a new live, whole-cell vaccine (ACE527) comprised of three attenuated ETEC strains [7]. Results from these studies were promising and PATH is planning to conduct further testing of ACE527 with and without co-administered adjuvant prior to entering a descending-age study in an ETEC-endemic country. The study is expected to launch in 2013.

Inactivated whole-cell ETEC vaccines also showed impressive progress. An inactivated ETEC vaccine composed of four ETEC strains engineered to overexpress CFA/I, CS3, CS5 and CS6 and supplemented with a LTB-CTB hybrid toxoid (Jan Holmgren, Goteborg University, Goteborg, Sweden) induced strong intestinal and serum antibody responses to these antigens in a mouse model, which could be significantly improved by administration with the double mutant heatlabile toxin mucosal adjuvant. In a human Phase I trial, an inactivated, overexpressing CFA/I strain and the new hybrid toxoid induced stronger intestinal (fecal IgA) or intestinal-derived antibody responses to CFA/I and LTB than the first-generation ETEC-inactivated vaccine.

While secondary analysis of a Phase II LT-patch transcutaneous immunization study demonstrated significant protection against ETEC-LT strains (ELT206) (Larry Ellingsworth, Intercell, MD, USA), the Phase III trials' primary endpoints (ELT301), reduction of incidence of all types of ETEC (defined as cases in which ETEC LT, LT/ST or ST toxins are detected in diarrheal stool samples) and/or all cause diarrhea (secondary endpoints) comparing the vaccine groups with the placebo group, were not met.

Also, a prototype conserved ETEC tip-adhesin (FTA)-based vaccine, delivered by transcutaneous immunization and intradermal routes was well tolerated and induced impressive levels of anti-tip adhesin ELISA and functional antibodies (Stephen Savarino, Naval Medical Research Center, MD, USA). Results from ongoing nonhuman primate studies demonstrated that intradermal immunization with FTA and mLT induced significant protection (84\%) against challenge with a fully virulent ETEC strain [6].

While targeting CFs antigens in ETEC vaccine development show very promising results, it should be kept in mind that these CFs antigens may undergo an antigenic mutations causing failure of CF-based ETEC vaccines. Additionally new emerging ETEC strains could hit under certain circumstances. Therefore looking for a conservative antigenic determinant in ETEC should be of high priorities. Selecting ST as a candidate for constructing a broad spectrum ETEC vaccine has been rationalized by its conservative structure in all ETEC strains, however this approach has been challenged by its haptenic and toxicity nature [5]. Therefore research efforts should be directed to overcome these challenges for constructing a non-toxigenic version of immunogenic ST. Such version is often referred to as an ST toxoid. The application of advanced genomic, proteomic and nanotechnologies as well as new vaccine delivery routes will pave the way to accomplish this goal.

\section{References}

1. World Health Organization. Enterotoxigenic Escherichia coli.

2. Rao MR, Abu-Elyazeed R, Savarino SJ, Naficy AB, Wierzba TF, et al. (2003) High disease burden of diarrhea due to enterotoxigenic escherichia coli among rural Egyptian infants and young children. J Clin Microbiol 41: 4862-4864.

3. Svennerholm AM, Steele D (2004) Microbial-gut interactions in health and disease. Progress in enteric vaccine development. Best Pract Res Clin Gastroenterol 18: 421-445.

4. Holmgren J, Svennerholm AM (1998) Vaccines against diarrheal disease Handbook of Experimental Pharmacology, Berlin: Springer 133: 291-328.

5. Walker RI, Steele D Aguado T, Ad Hoc ETEC, Technical Expert Committee (2007) Analysis of strategies to successfully vaccinate infants in developing countries against enterotoxigenic E. coli (ETEC) disease. Vaccine 25: 25452566.

${ }^{*}$ Corresponding author: Nasr-Eldin Mohamed M. Aref, Department of Animal Medicine, Faculty of Veterinary Medicine, Assiut University, Assiut-71526, Egypt, Tel: +0160024252; E-mail: nasreldeen.aref@vet.au.edu.eg

Received August 28, 2012; Accepted September 04, 2012; Published September 08, 2012

Citation: Aref NEMM (2012) ETEC Vaccine between Reality and Expectations. J Vaccines Vaccin 3:e110. doi:10.4172/2157-7560.1000e110

Copyright: (c) 2012 Aref NEMM. This is an open-access article distributed under the terms of the Creative Commons Attribution License, which permits unrestricted use, distribution, and reproduction in any medium, provided the original author and source are credited. 
6. Steele D, Riddle M, van de Verg $L$, Bourgeois $L$ (2012) Vaccines for enteric diseases: a meeting summary. Expert Rev Vaccines 11: 407-409.

7. Clayton Harro, David Sack, Louis Bourgeois A, Walker R, Barbara DeNearing, et al. (2011) A combination vaccine consisting of three live attenuated enterotoxigenic Escherichia coli strains expressing a range of colonization factors and heat-labile toxin subunit $B$ is well tolerated and immunogenic in a placebo-controlled double-blind Phase I trial in healthy adults. Clin Vaccine Immunol 18: 2118-2127. 